\title{
Bio-structural monitoring of bone mineral alterations through electromechanical impedance measurements of a Piezo-device joined to a tooth
}

\author{
Hector A. Tinoco ${ }^{1,2,3}$. Carlos I. Cardona ${ }^{1}$ - Maribel L. F. Marín-Berrio ${ }^{4} \cdot$ Juliana García-Grisales ${ }^{4}$. Juan P. Gomez ${ }^{4}$. \\ Samuel I. Roldan ${ }^{5} \cdot$ Fabio M. Peña ${ }^{1} \cdot$ Adam Brinek $^{3} \cdot$ Dominika Kalasová $^{3} \cdot$ Jozef Kaiser $^{3} \cdot$ Tomas Zikmund $^{3}$
}

Received: 22 May 2020 / Revised: 3 August 2020 / Accepted: 9 September 2020 / Published online: 20 September 2020

(c) Korean Society of Medical and Biological Engineering 2020, corrected publication 2020

\begin{abstract}
Bone presents different systemic functionalities as calcium phosphate reservoir, organ protection, among others. For that reason, the bone health conditions are essential to keep in equilibrium the metabolism of several body systems. Different technologies exist to diagnose bone conditions with invasive methods based on ionizing radiation. Therefore, there is a challenge to develop new ways to evaluate bone alterations in a noninvasive form. This study shows the assessment of a piezo-actuated device acting on a human tooth for the bio-monitoring of bone alterations. The bone diagnosis is performed by applying the electromechanical impedance technique (EMI), commonly used in structural health monitoring. For the experimental tests, five bone samples were prepared, and one was chosen as the monitoring. All samples were put in a decalcifying substance (TBD1 acid-base) at different times to emulate localized bone mineral alterations. Bone reductions were computed by using $\mathrm{X}$-ray micro-computed tomography analyzing the morphometry. Electrical resistance measurements (piezo-device) were taken for the monitoring specimen meanwhile it was partially decalcified during 8520 seconds. In the frequency spectrum, several observation windows showed that the bone alterations gradually changed the electrical resistance signals which were quantified statistically. Results evidenced that the bone density changes are correlated with the electrical resistance measurements; these changes presented an exponential behavior as much as in the calculated index, and bone mineral reduction. The results demonstrated that bone alterations exhibit linear dependence with the computed statistical indexes. This result confirms that it is possible to observe the bone changes from the teeth as a future application.
\end{abstract}

Keywords Bone mass reduction $\cdot$ Bone mineral alterations $\cdot$ Bio-monitoring $\cdot$ Spectroscopy $\cdot$ Electromechanical impedance technique $\cdot$ Bone mineral density $\cdot$ Micro-CT $\cdot$ Piezo-device $\cdot$ Structural health monitoring

Hector A. Tinoco

htinoco@autonoma.edu.co

1 Experimental and Computational Mechanics Laboratory, Universidad Autónoma de Manizales. Antigua Estación del Ferrocarril, Edificio fundadores, C.P. 170001 Manizales, Colombia

2 Institute of Physics of Materials, Sciences Academy of Czech Republic, Žižkova 22, 61662 Brno, Czech Republic

3 Central European Institute of Technology - Brno University of Technology, 648/125 Brno, Czech Republic

4 Department of Health, Universidad Autónoma de Manizales, Antigua estación del Ferrocarril, Edificio fundadores, C.P. 170001 Manizales, Colombia

5 Centro de Innovación Roldan, Cl 4 Sur 43 Aa 26, Medellin-Antioquia, Colombia

\section{Introduction}

Gradual alterations or reduction of bone mineral density (BMD) may occur due to different pathologic causes including systemic changes such as osteoporosis. In 2013, the united nations reported more than 22 million women and 5.5 million men with osteoporosis. In the same year, the number of fragility fractures caused approximately 37 billion Euros of additional financial burden to the local health system [1]. Using the World Health Organization (WHO) criteria, 30\% of postmenopausal Caucasian women have osteoporosis at the hip, lumbar spine or distal forearm [2]. The problem, in terms of prevalence, seems as severe as coronary heart disease, since the estimated lifetime risk for a hip, wrist or vertebral fracture is about $35 \%$ in developed countries and at the age of 50, in a Caucasian female's the risk of having an 
osteoporotic fracture is estimated in 46 years old with a 53\% of risk, compared to men is about $13-21 \%[3,4])$.

In order to improve the quality of life affected negatively by the impact of loss in bone mineral density (BMD), early detection of systemic reduction in BMD should be achieved. Current technologies used to measure BMD require invasive methods due to ionizing radiation. Diagnosis for the variations of BMD may be detected with techniques that range from the least injurious conventional digital radiographs [5] to the high ionizing radiation emitted by micro-CT [6]. Although computerized Radiogrammetry (CRG), abdominal Computed Tomography (CT), Quantitative Computed Tomography (QCT) [7], Radiographic Photodensitometry (RP), Dual-energy Photon Absorptiometry [8] and Dualenergy X-ray absorptiometry (DEXA) that is the most widely used for osteoporosis screening [9]. Despite there is evidence regarding the inter, and intraindividual variability of BMD, investigations using DEXA have suggested a correlation between BMD values obtained from maxillary bones with those acquired from sites recommended by WHO for osteoporosis screening, for example lumbar vertebras and femur [10]. López-López et al. [11] concluded that the panoramic X-Ray could be an important tool to do early detection of osteoporosis, but the periapical X-ray showed low correlations with DEXA. The evidence shows in a positive way that several methods and techniques are used successfully in the bone diagnosis; but these are limited by aspect as each country's health system, costs, accessibility, and repeatability. Therefore, it seems reasonable to explore new ways of detecting BMD variations with non-conventional techniques.

During the last few years, structural health monitoring (SHM) techniques have permitted to explore several nondestructive evaluation methodologies with piezoelectric transducers in structures that require continuous monitoring, such as civil structures, aircraft structures, and industrial infrastructures [12-17]. In SHM field, electromechanical impedance (EMI) technique has shown to be useful in the detection of structural changes by applying high-frequency vibrations (usually $>5 \mathrm{kHz}$ ) with piezoelectric transducers. These act as monitoring elements that can be activated as sensors and actuators when joined to a host structure. When the host structure presents variations in its initial conditions (mass and stiffness variations), electrical impedance measurements can detect the mechanical impedance changes due to the electromechanical coupling effect demonstrated initially by Liang et al. [18] and posteriorly applied by other authors [16, 19-21]. As such, EMI technique has also been applied to biological structures for monitoring their mechanical properties related intrinsically with different biological phenomena. Bhalla and Suresh [22], Khan et al. [23], Srivastava et al. [24] used piezoelectric transducers as biosensors to monitor bone conditions through measuring the electrical impedance of a piezo transducer. These studies showed that detect bio-structural variations such as; cracks, total fractures, a healing process, and osteoporosis, could be possible in the future. Srivastava et al. [25] and Srivastava and Bhalla [26] proposed an experimental design for studying numerically the structural state of bones measuring in vitro bone specimens and an in vivo subject. The procedures were carried out using a piezo-patch coupled to an aluminum beam, which in turn is fixed to a removable assembly in the study specimens. The acquired electrical conductance measurements were taken in a live human arm, an artificial tissue-skin bone assembly, and one measurement acquired conventionally of a piezo-sensor directly bonded to a bare bone. However, these results were not applicable due to the fact that it is not possible to manipulate bone conditions in living humans.

Several SHM studies have implemented the EMI technique for the bio-structural diagnosis in search of biomedical applications. For instance, Tabrizi et al. [27] and Ribolla et al. [28] used numerical models and experiments to demonstrate the viability of using piezo-transducers in a dental implant to evaluate the stability condition after insertion. Ponder et al. [29] presents experimental tests where electrical impedance measurements are taken for assessing a bone-implant interaction that emulates a total knee replacement case. Results showed that the progressive induced damage on the implant was detected with the electrical sensitivity of the piezo transducers. Other authors have developed a novel approach for monitoring structural variations by using human teeth as biological probes embedded in different substrates [30]. Sine sweep vibrations between 5 and $10 \mathrm{kHz}$ were applied using a piezo-device mounted on an orthodontic bracket bonded in a dental crown, the mechanical response of the substrate was quantified from the electrical resistance of the piezomaterial. Results showed that the materials of the substrate were differentiated. Moreover, Tinoco et al. [31] developed a piezo-device to detect Young's modulus changes of three different substrates by means of electrical resistance measurements $(1-20 \mathrm{kHz})$ obtained from a tooth-bracketdevice system. The study allowed the authors to conclude that different types of bio-structures could be monitored since electrical resistance signals correlated directly with the substrate elasticity.

Other approaches have been explored for monitoring bio-structures using bioimpedance analysis. It consists in measuring the electrical resistivity by applying low current in the specimen to be monitored to determine different conditions of the biomaterial, for example, the electrical resistivity of the bone tissue as evidenced Xie et al. [32] and Pietrobelli et al. [33]. For instance, Balmer et al. [34] explored a manner to characterize bone properties in vivo, measuring the electrical resistivity of sheep's mastoid bone with two clamped electrodes at low frequencies $1 \mathrm{kHz}$ to 
predict the quantity of bone material between electrodes. Besides, Kimel-Naor et al. [35] developed a parametric electrical impedance tomography tool, combining computational numeric algorithms with applied in vivo measurements taken in a three-dimensional scanned man pelvis model to study BMD alterations related with osteoporosis. However, using these methods, the electric resistivity is measured connecting electrodes directly in the bone sample. Moreover, these approaches employ an invasive way to measure due to electricity current passes through the bone, and it requires installing systems that have contact with the bone. Unlike those methods, current EMI based methods applied in structural health monitoring consist of propagating mechanical vibrations to monitor the mechanical impedance changes through electrical impedance measurements usually read by piezoelectric transducers linked to a test structure $[16,36]$.

The present study aims to develop a noninvasive methodology for the bio-monitoring of bone structural alterations by using the EMI technique. A bone tooth system is proposed to recreate a portion of the human maxillary bone. The exposed method applies mechanical vibrations through teeth into the bone. In this case, a piezo-device sensor is integrated into a bone specimen through a human tooth, which operates as a natural probe for the transmission of mechanical vibrations towards the bone tissue. The direct contact between the sensor and the bone sample is avoided using this method. Given these conditions, the electrical impedance is measured over the device that operates as an actuator and sensor, respectively. Results evidence the bone density changes during the time are read by the electrical resistance measurements; this allowed to observe the sensitivity of the EMI technique. The effects of bone alterations presented an exponential behavior in the calculated indexes and the estimated quantities of mineral density loss. In this study, it was demonstrated that measure bone alterations from the teeth could be a less invasive method for diagnosing the maxillary bone.

\section{Materials and methods}

\subsection{Bone samples preparation}

\subsubsection{Bone samples preparation protocol of a tooth and artificial periodontal ligament (PDL)}

In order to obtain the required size and geometry of the trabecular bone samples, roughly rectangular bone segments were cut from the central portion of a bovine hip bone obtained in a local butcher shop. This bone was selected from a bull hip bone since it is composed of a higher proportion of trabecular bone. Initial cuttings were made using a water-cooled mechanical circular saw, and final cuttings were made to achieve bone samples in regular cuboids using a milling machine. Bone segments were submitted to a first cleansing procedure for initial removal of soft tissues, in which the bone fragments were submerged in $70^{\circ} \mathrm{C}$ water and subsequently cleaned with a scalpel and scrubbed with a conventional toothbrush, three times a day for 1 week. More thorough removal of inter-trabecular residues (bone marrow, blood vessels) required chemical decomposition of different tissues, especially marrow adipose tissue that was not affected by initial cleansing. This subsequent cleansing procedure was performed using a mixture of dishwashing detergent and water. The soap used has tensio-active agents in order to eliminate the adipose tissue of a surface by the emulsifying power to produce colloidal dispersions of fat in an aqueous medium. Then, bone samples were submerged in the soapy solution that was changed three times a day for $4 \mathrm{r}$ weeks at $70^{\circ} \mathrm{C}$. Once the initial bone segments were sufficiently cleaned, the final geometric configuration of bone samples was obtained using a water-cooled circular saw, producing five rectangular bone samples.

Once bone samples were cut, an additional submersion in the detergent-based cleaning solution was performed to assure a deeper elimination of inter-trabecular residues. After testing different substances for the final cleansing procedure, the best results were obtained with 24-h submersion in $4 \%$ hydrogen peroxide, followed by abundant rinsing with water and drying at room temperature for $5 \mathrm{~h}$ and subsequent drying at $50^{\circ} \mathrm{C}$ to produce the definitive rectangular bone samples (see Fig. 1d) as has been similarly developed by Rodríguez-Palomo and Ramírez-zamora.[37]. Procedures for cleansing and preparing bone specimens, typically consist on boiling the samples in water, then removing tissues and finally using detergents to remove the remaining tissue trapped, as mentioned by Mairs et al. [38], that studied the effectiveness of soaps for the eliminations of soft tissue in animal bone specimens. Finally, the dimensions of the prepared samples are shown in Table 1.

The alveolar cavity was manufactured by mechanical drilling using a conic bit which had the same geometry of the tooth root with the artificial periodontal ligament. Drilling depth and diameter were $14 \mathrm{~mm}$ and $6 \mathrm{~mm}$, respectively.

\subsubsection{Artificial PDL preparation}

The importance of the PDL in the biomechanics of the toothbone assembly suggests the elaboration of a coherent substitute in tooth-bone assembly. For this purpose, the supporting structures contained in a portion of a maxillary tooth system were manufactured with a $0.3 \mathrm{~mm}$ layer of Polyvinyl Siloxane (PVS). The artificial PDL was made aiming to model a more realistic bone-tooth interaction where stress distribution patterns are affected in the PDL $[39,40]$. The artificial 


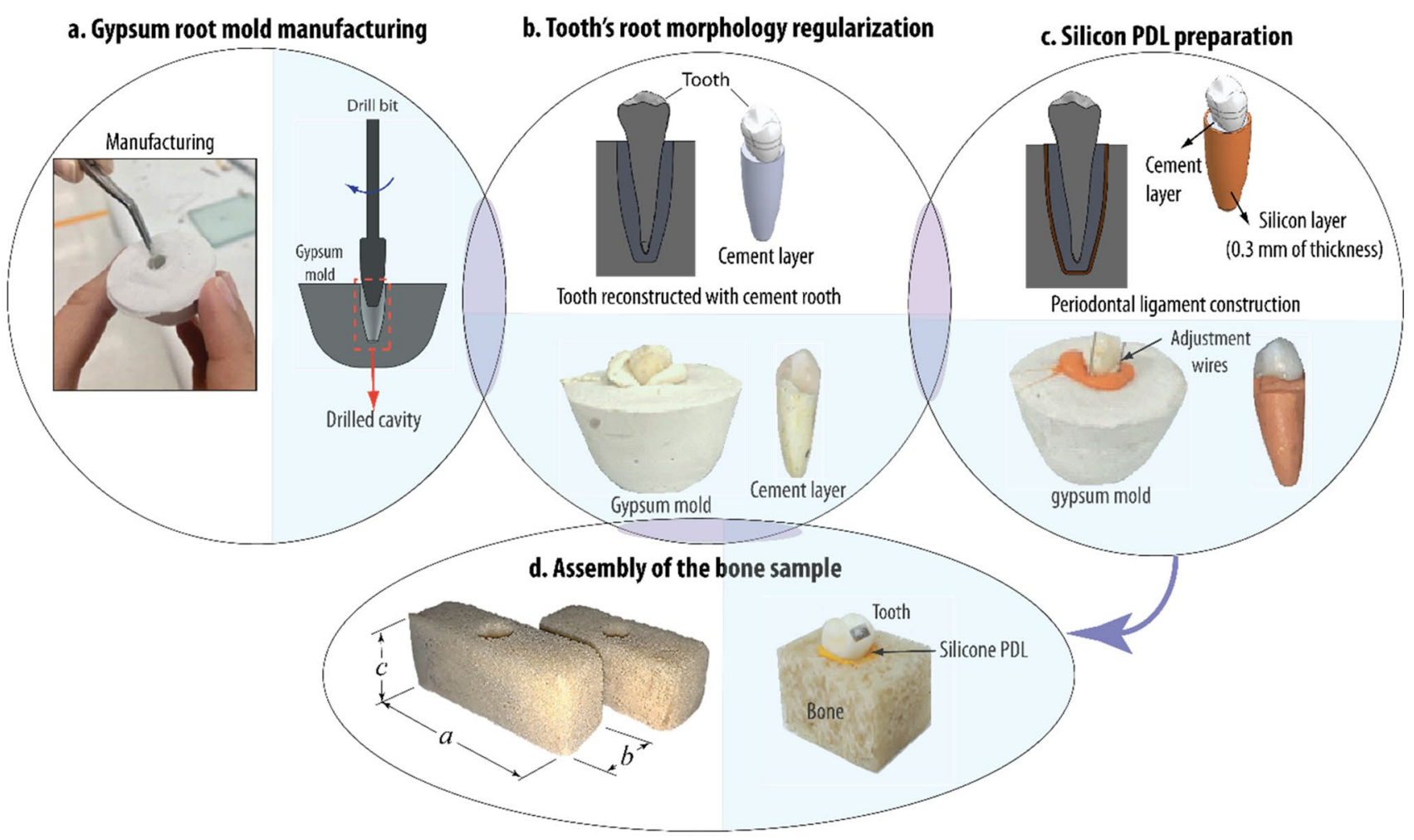

Fig. 1 Artificial PDL manufacturing procedure and bone-tooth assembly scheme

Table 1 Principal dimensions and mass of the bone samples (see Fig. 1d)

\begin{tabular}{lllll}
\hline Sample & $\mathrm{a}(\mathrm{mm})$ & $\mathrm{b}(\mathrm{mm})$ & $\mathrm{c}(\mathrm{mm})$ & Initial weight $(\mathrm{g})$ \\
\hline 1 & 19.2 & 18.91 & 12.8 & 3.68866 \\
2 & 19 & 18.75 & 11.8 & 1.70728 \\
3 & 19 & 19.12 & 12.75 & 3.3934 \\
4 & 19.06 & 18.55 & 10.96 & 3.3934 \\
5 & 18.85 & 12.33 & 19.13 & 1.48976 \\
\hline
\end{tabular}

(PDL) manufacturing scheme is depicted in Fig. 1, in which the following stages were developed:

- Gypsum root mold manufacturing With the same bit used to drill the artificial alveolus in the bone samples, an equivalent perforation was made in a block of dental cast gypsum. The resulting cavity was isolated with petroleum jelly to avoid adherence to phosphate cement as Fig. 1a shows.

- Tooth root morphology regularization The entire surface of the root was treated with $37 \%$ phosphoric acid for $60 \mathrm{~s}$ as an etching agent to improve adherence of zinc phosphate cement. After filling the root mold cavity with previously prepared zinc phosphate cement with putty-like consistency, the tooth is forcefully inserted until contact with the bottom of the cavity. After $3 \mathrm{~h}$ of setting the tooth, it was removed from the mold, as illustrated in Fig. 1b. The excess cement mixture that extruded at the cervical limit was carefully eliminated, providing a root morphology that coincides with the morphology of the artificial alveolus.

- Artificial PDL conformation Using the gypsum mold, three segments (length of 1 in) of 0.012 in (diameter) NiTi wires were inserted every $120^{\circ}$ into the mold cavity before being filled with light-body PVS elastomer. The previously prepared tooth was inserted into the hollow until contact with the NiTi wires, that were assured and left to cure for $1 \mathrm{~h}$. This technique provided a uniform PVS thickness resembling PDL average thickness that is $0.25-0.3 \mathrm{~mm}$ [41]

- Assembly of the bone sample After curing, NiTi wires were removed, gypsum cast destroyed and artificial PDL structure obtained without risk of PVS tearing. Then, tooth-PDL assembly was inserted and stabilized into the alveolus of the previously manufactured bone sample.

\subsection{Monitoring of bone mineral alterations through EMI technique}

In this section is described a methodology to assess bone structural alterations through a piezo-actuated device, and 
the application of EMI technique [16, 42, 43]. Liang et al. [18] introduced the principles of the EMI technique developing a simple model for the interaction of a piezoelectric sheet with its host structure. The authors explained the effects of electromechanical coupling as a function of the mechanical impedances of both structures. The advantage of this approach is that the mechanical properties are related directly with the electrical parameters of the active materials (that act as sensor and actuator), which can be piezoelectric, electrostrictive, and magnetostrictive. In the last years, many studies have used and confirmed these fundamentals in several engineering applications as mentioned in the introduction. In summary, the one-dimensional model presented by Liang et al. [18] is written as follows permittivity of the piezoelectric material at constant stress. $\eta$ and $\delta$ denote both mechanical loss and dielectric loss factors, $k$ is the wave number. $Z_{p}^{M}(\omega)$ and $Z_{T p}^{M}(\omega)$ represent the mechanical impedances of the piezoelectric sheet and the host structure.

Figure 2 shows an experimental scheme that includes a bone structure joined to a piezo-actuated device bonded to a tooth. The main idea is to monitor the bone structural conditions through mechanical vibrations applied with the electromechanical device, which was developed by Tinoco et al. [31]. The authors describe all dynamics characteristics, and they mention the potential applications in the context of bio-structural analysis.

The proposed main idea in Fig. 2, subsequently will

$\frac{1}{Z_{P}^{E}(\omega)}=\frac{2 \omega j w_{p}}{h_{p}}\left[e_{33}-d_{31}^{2} \bar{y}^{E}+\left(\frac{Z_{p}^{M}(\omega)}{Z_{p}^{M}(\omega)+Z_{T p}^{M}(\omega)}\right) d_{31}^{2} \bar{y}^{E}\left(\frac{\tan \left(k l_{p}\right)}{k l_{p}}\right)\right]$,

where $h_{p}$ represents the thickness, $l_{p}$ the length, $w_{p}$ the width of a piezoelectric sheet, $d_{31}$ is the piezoelectric strain coefficient corresponding to $x(1)-z(3)$ coordinates, $\bar{y}^{E}=y^{E}(1+\eta)$ is the complex Young's modulus at a constant electric field and $\bar{e}^{\sigma}=e^{\sigma}(1+\delta)$ is the complex electric help to demonstrate that the bone alterations can be monitored using the teeth as probes, since if the bone structure changes $\left(Z_{s}^{M}(\omega)\right)$, the new vibratory conditions (changes in $\left.Z_{P a}^{M}(\omega)\right)$ will modify the electrical impedance $Z_{P}^{E}(\omega)$ of the piezoelectric patch, as evidenced by Tinoco et al.

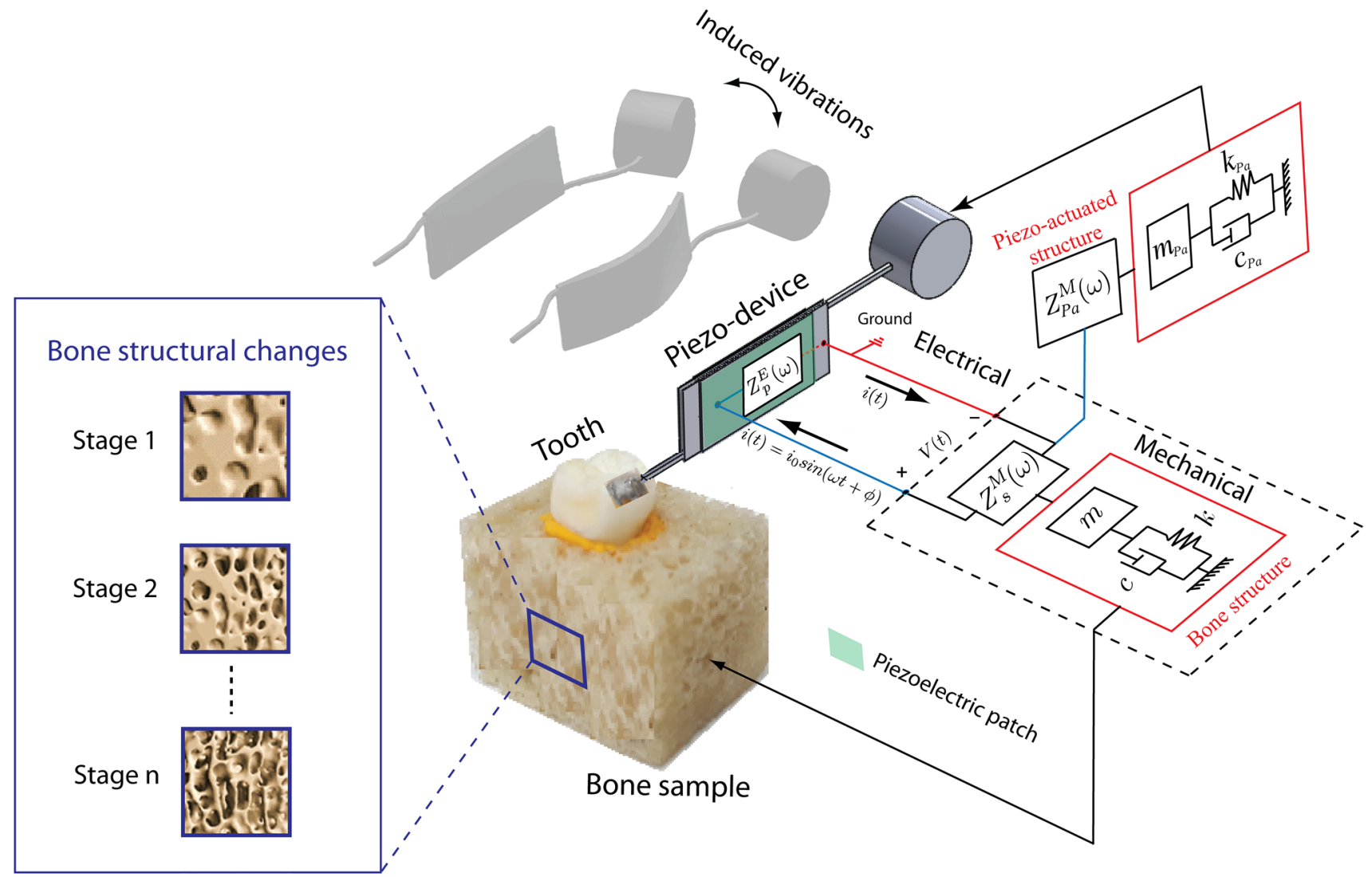

Fig. 2 Monitoring of bone structural changes through a piezo-device applying EMI technique 
[31] and Barco et al. [44]. Bone alterations are assumed as the changes in BMD, which affect its structural condition and the natural quality. Therefore, a decalcification substance will be used to produce density losses during the experiments,

Piezoelectric transducers (PT) present electrical properties due to the electromechanical phenomenon. If these are connected to an electrical circuit, PT will be an active part of the circuit. In principle, a piezo-transducer is represented by a combination of resistance and capacitance as discussed by Sirohi and Chopra [45], which indicates that there is no inductance in a piezo-transducer. Following this idea, the electrical impedance of a PT is represented by

$Z_{P}^{E}(\omega)=\frac{i_{0}(\omega)}{V_{0}(\omega)}=R(\omega)+X_{c}(\omega) j$

where $\omega$ is the frequency, $i_{0}(\omega)$ and $V_{0}(\omega)$ are the electrical current and the electrical potential. $R(\omega)$ and $X_{c}(\omega)$ are the electrical resistance and the capacitive reactance. Electrical impedance variations can be quantified using indexes calculated by comparing two electrical impedance signals; a baseline and a monitoring signal. The baseline is a signal taken when the structure presents optimal conditions, and another one is the signal measured as a control parameter. The quantitative comparisons are performed by the computations of several statistical indices, among these; root means square deviation (RMSD), correlation coefficient deviation metric (CCDM), mean absolute percentage deviation (MAPD) and others reported in the literature [46-48]. In this study, an

(a)

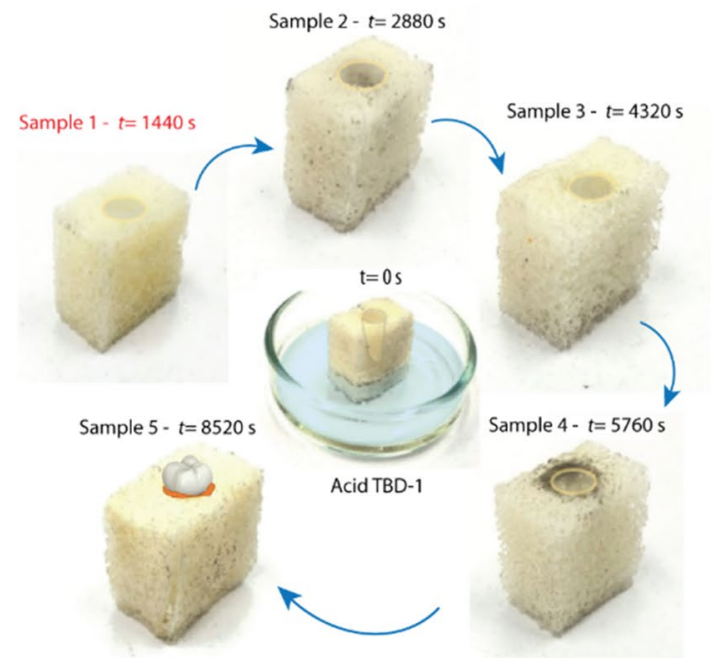

RMSD index is calculated for the variations of electrical resistance, which is defined as follows

$\varphi=\sqrt{\frac{1}{n} \sum_{i=1}^{n}\left(\frac{R_{i(M)}-R_{i(\text { Ref })}}{R_{i(\text { Ref })}}\right)^{2}} \times 100$.

where $R_{i(M)}$ is the monitoring signal and $R_{i(\operatorname{Ref})}$ corresponds to the values of the baseline. In other words, the quantification of the index reflects the statistical differences between a healthy and modified structure. IThe absolute values of the index should be correlated with the physical parameter to represent any physical meaning since its values are determined in percentages.

\subsection{Experimental setup for the monitoring of induced bone mineral alterations}

The working hypothesis for the proposed bio-monitoring procedure is that the piezo-device can detect small bone structural changes, as explained in Sect. 2.2. Therefore, an experiment was designed to induce gradual bone mineral reduction using a decalcification protocol that induced a pathologic scenario of decreased BMD. The experimental design was proposed with five samples; in which four samples were defined as replicas (without tooth), and the fifth sample was chosen as a monitoring structure (with embedded tooth). All samples were immersed partially in $3 \mathrm{ml}$ decalcifying acid TBD-1 (Thermo-Shandon SA, Pittsburgh, PA, USA) using a Petri box as its container. TBD-1 is a typical decalcification substance, as mentioned by Calvo-Guirado et al. [49]. A description of the order of

Fig. 3 a Exposure times of TBD-1 for the bone samples. b Experimental setup to measure the electrical resistance of the piezo-device on the fifth sample 
the samples into the experimental development is detailed in Fig. 3a.

In the experiments, we consider that soft tissues would not perturb the electrical impedance signals for the following reasons: the stiffness relation between soft tissue and bone is predominated by bone tissue. Therefore, we assume the following hypothesis. Soft tissue could affect the system damping factor if there was a considerable quantity of it, and as a consequence, the mechanical vibrations could be attenuated but not the main mechanical resonances. It means that the whole mechanical system would not present a change that will affect the proposed methodology.

For carrying out the experiments with the bone samples, the piezo-device was integrated into the fifth sample by coupling it to a premolar tooth utilizing a bracket. An impedance analyzer equipment (E4990, Agilent, Palo Alto, CA, USA) was configured with a sweep setup signal between $5-50 \mathrm{kHz}$ and 1601 resolution points to measure the electrical impedance of the piezo-device as shown in Fig. $3 b$.

The first sample was exposed to the acid solution during $1440 \mathrm{~s}$; the second one to $2880 \mathrm{~s}$, the third to $4320 \mathrm{~s}$, the fourth to $5760 \mathrm{~s}$, and the fifth one to $8520 \mathrm{~s}$ (full time), Fig. 3a. It is important to denote that only the fifth sample was prepared with an embedded tooth and an artificial PDL layer. The experiment is designed to reconstruct the progressive bone alterations caused by TBD-1 effects of sample 5 in all-time domain with the characterization of the remaining (samples 1 to 4). The main intention of replica samples is aiming to analyze the progressive bone alterations caused by TBD- 1 effects.

In the monitoring specimen (sample 5), a reference measurement (original bone) was taken without the presence of TBD-1 substance, and fourteen impedance measurements were read until the total exposure time $(8520 \mathrm{~s})$. The decalcifying volume of the samples was defined on the base of the sample that corresponds with the surface opposite to the alveolar cavity, and these characteristics are listed in Table 2.

Table 2 Main dimensional features of the bone samples determined for the experimental process

\begin{tabular}{lllll}
\hline Sample & $\begin{array}{l}\text { Submerged } \\
\text { height }(\mathrm{mm})\end{array}$ & $\begin{array}{l}\text { Sample vol- } \\
\text { ume }\left(\mathrm{mm}^{3}\right)\end{array}$ & $\begin{array}{l}\text { Alveolus } \\
\text { volume } \\
\left(\mathrm{mm}^{3}\right)\end{array}$ & $\begin{array}{l}\text { Submerged } \\
\text { volume }\left(\mathrm{mm}^{3}\right)\end{array}$ \\
\hline 1 & 1.66 & 4419.75 & 254.46 & 407.96 \\
2 & 1.68 & 3976.18 & 254.46 & 376.65 \\
3 & 1.71 & 4404.25 & 254.46 & 414.25 \\
4 & 1.70 & 3647.48 & 254.46 & 355.12 \\
5 & 1.64 & 4218.63 & 254.46 & 591.38 \\
\hline
\end{tabular}

\section{Results and discussion}

\subsection{Morphometrical characteristics of altered bone samples determined by Micro-CT analysis}

In this section are discussed some aspects related to the morphometrical characteristics of the bone samples after carrying out the experiment described in Sect. 2.3. Figure 4a represents the images obtained with a ZEISS Axio Zoom V16 optical microscope that reveals the microarchitecture of the bone samples (from 2 to 5) in the demarked locations at the plane A-A. According to the images, morphometrical differences among the samples are observed due to these were extracted from different bovine hip bones. In samples 4 and 5, trabecular walls are distinguished by the contrast of the porosity (superficially clean), on the contrary case, samples 2 and 3 seem to be denser. In order to provide a precise morphometrical description [50], a Heliscan micro-computed tomography equipment (Thermofisher Scientific) was used. The bone samples were scanned in one measurement with helical space filling trajectory and iterative reconstruction with X-ray tube voltage $90 \mathrm{kV}$, and lineal voxel size of $13.9 \mu \mathrm{m}$. Section planes were defined, aiming to observe the inner structure and morphology of each sample, transversally (plane A-A) and vertically (top plane), as shown in Fig. 4b. It is observed that sample 2 contains more quantity of cancellous structure, followed by the sample 3, 5 , and 4 . We point out, particularly, that sample 3 includes not only trabecular bone but also residual organic material that was not removed during the cleaning process. It can be observed comparing the contrast of the segmented image, where white regions represent the trabecular bone, and gray regions correspond with uncleaned material. Furthermore, the images evidenced that the construction of the alveolar cavity seems homogeneous in all samples from a morphological point of view; it reproduces the natural tooth socket.

For the micro-CT analysis, a grey value threshold was established to extract the bone structure through a color segmentation process in the samples. It was carried out to differentiate the pristine structure (white bone) with the altered bone region. Besides, as a result of the decalcification in all samples, the degraded areas can be observed in the bottom part (dark gray), as illustrated in the cross-section $\mathrm{A}-\mathrm{A}$. These regions are more visible in samples four and five because these were exposed more time in the decalcifying process. For providing a qualitative comparison of the bone alterations based on visualization, sample 1 (1440 s) and 5 (8520 s) are compared, as shown in Fig. 5a. Dark blue regions are those modified by the acid TBD-1, and the blue ones correspond with the healthy cancellous bone structure. It is observed that the induced alterations were not uniform at the base of both samples due to the porosity 
Fig. 4 a Bone microstructure for the samples 2 until 5. b Cross-sections of the samples (2 until 5) obtained by micro-CT
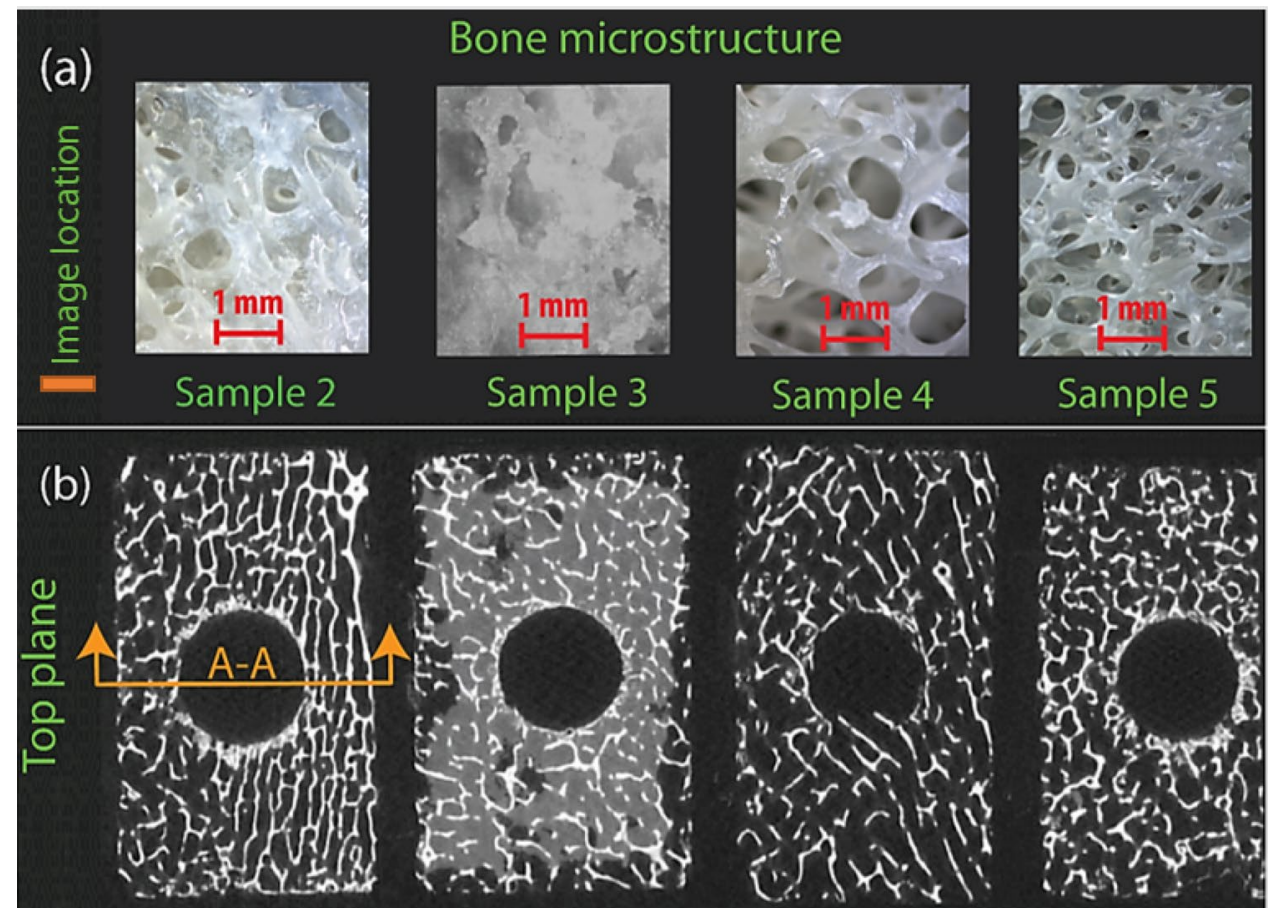

Y" ch

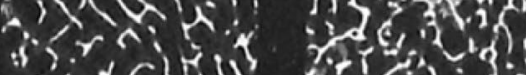

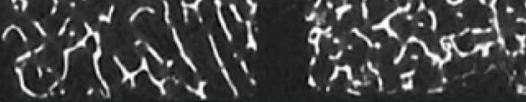

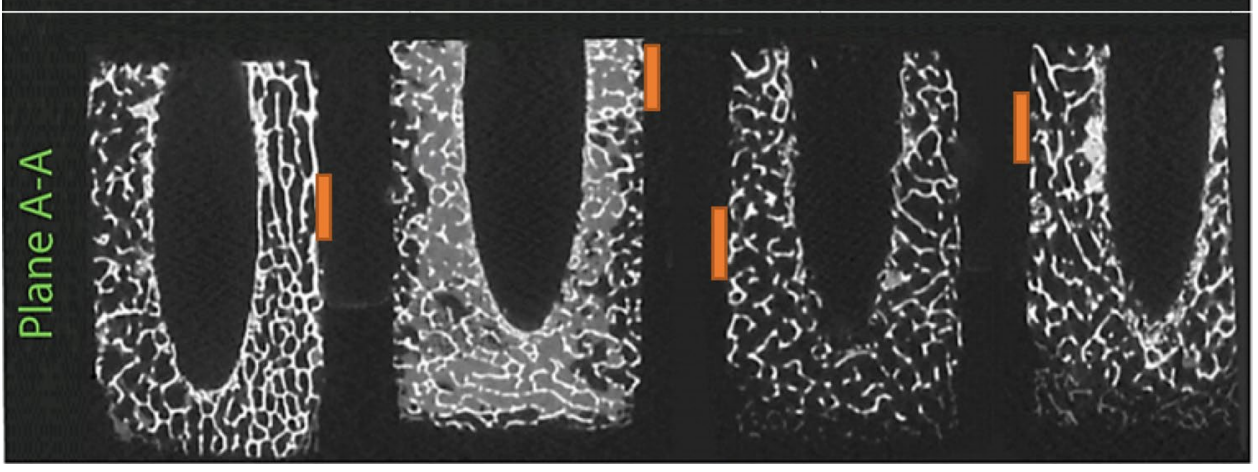

Fig. 5 a 3D rendering for the CT data of samples 1 and 5 . b Determination of analysis regions in bone segmentation, sample 5

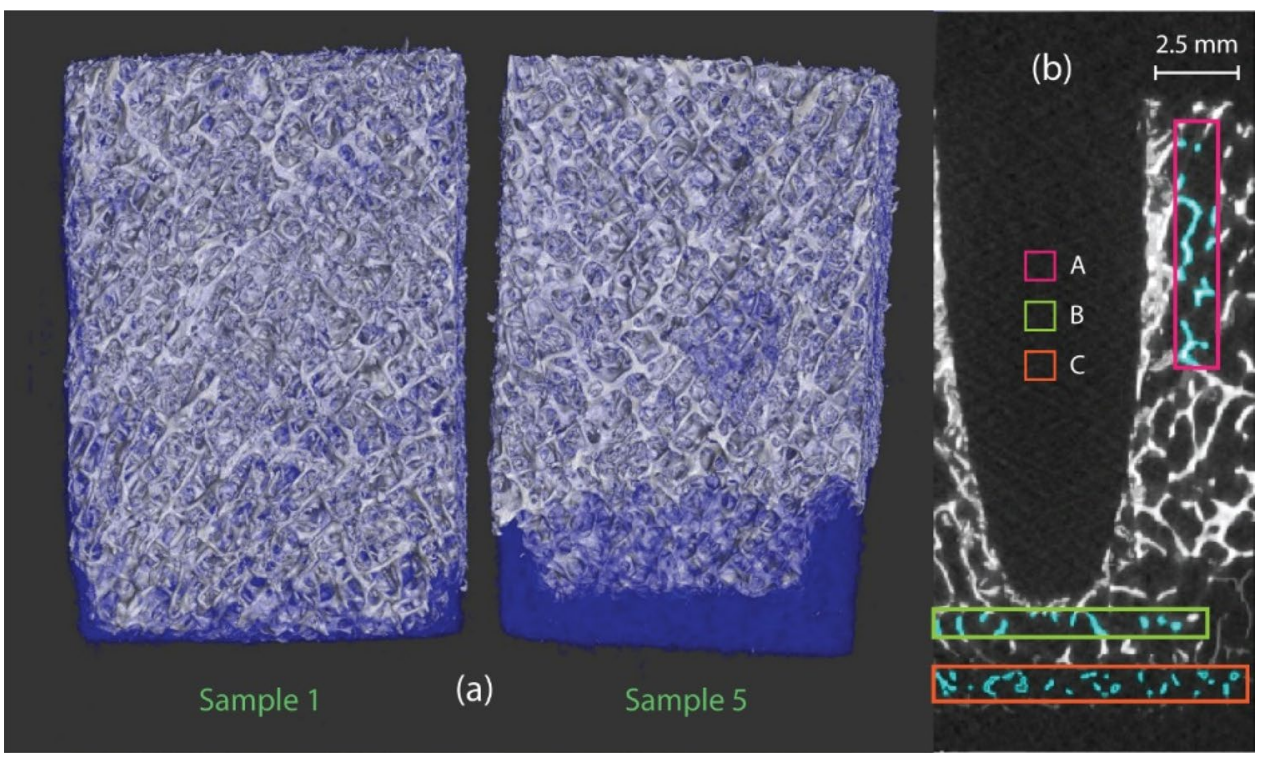


Table 3 Results for the micro-CT Analysis

\begin{tabular}{lrllll}
\hline $\begin{array}{l}\text { Analy- } \\
\text { sis } \\
\text { region }\end{array}$ & $\begin{array}{l}\text { Total } \\
\text { volume } \\
\left(\mathrm{mm}^{3}\right)\end{array}$ & $\begin{array}{l}\text { Bone } \\
\text { volume } \\
\left(\mathrm{mm}^{3}\right)\end{array}$ & BFV $(\%)$ & $\begin{array}{l}\text { Trabecular } \\
\text { thickness } \\
(\mu \mathrm{m})\end{array}$ & $\begin{array}{l}\text { Thickness } \\
\text { deviation } \\
(\mu \mathrm{m})\end{array}$ \\
\hline 1A & 118 & 28 & 24 & 231 & 64 \\
1B & 54 & 11 & 21 & 221 & 63 \\
1C & 62 & 12 & 20 & - & - \\
2A & 118 & 31 & 26 & 185 & 66 \\
2B & 54 & 13 & 24 & 148 & 42 \\
2C & 43 & 10 & 22 & - & - \\
3A & 118 & 28 & 24 & 194 & 61 \\
3B & 54 & 14 & 25 & 176 & 52 \\
3C & 98 & 22 & 23 & - & - \\
4A & 118 & 15 & 13 & 149 & 50 \\
4B & 54 & 5 & 10 & 137 & 45 \\
4C & 98 & 6 & 6 & - & - \\
5A & 118 & 20 & 17 & 165 & 50 \\
5B & 54 & 8 & 15 & 148 & 42 \\
5C & 98 & 9 & 10 & - & - \\
\hline
\end{tabular}

Table 4 Mass measurements before and after the decalcifying process

\begin{tabular}{llllc}
\hline Sample & $\begin{array}{l}\text { Exposure } \\
\text { time (s) }\end{array}$ & Initial mass (g) & Final mass (g) & \% Mass loss \\
\hline 1 & 1440 & 3.6887 & 3.6483 & 1.0936 \\
2 & 2880 & 1.7073 & 1.6236 & 4.9014 \\
3 & 4320 & 3.3934 & 3.3418 & 1.5218 \\
4 & 5760 & 1.0433 & 0.9471 & 9.2192 \\
5 & 8520 & 1.4898 & 1.3223 & 11.2381 \\
\hline
\end{tabular}

of the trabecular morphology, which probably caused that the acid flowed more in some regions than in others. For the quantification of the morphometric parameters, some regions were selected for this purpose on the samples. The objective is to compare three regions in each sample, and these are called region A (upper), region B (low alveolar cavity), and region $\mathrm{C}$ (bottom of the sample); these were marked and detailed in Fig. 5b. It is important to clarify that the volume of the regions was standardized for zones $\mathrm{A}$ and $\mathrm{B}$ as shown in Table 3. Zone $\mathrm{C}$ could not be fixed since the parameters that defined the bone structure were variable for the characterization.

The discrimination of the zones A, B and, C provide comparison points of the induced morphologic changes after the alterations introduced by the acid. Zone A was selected away from the acid action, and this region was not modified. Zone $\mathrm{B}$ was defined closer to the acid action, but according to the micro CT analysis, this region was not altered either. Finally, Zone $\mathrm{C}$ was determined as the region directly changed by the acid and chosen to study the incidence of it in the bone microarchitecture. Collected data from the defined zones for each sample are denoted as "number of the sample, zone letter"; for instance, zone 1A corresponds to the sample 1 in the zone A. All the data of the measurements are listed in Table 3. The determined morphometric variables were the following; total volume, bone volume, bone fraction volume (BFV), trabecular thickness, and its deviation.

In Table 4 are reported the reduction values of bone mass during the decalcifying process since initial and final mass measurements were taken for all samples. It is seen that each sample presented different values of variation in the bone mass without any clear trend. It indicates that there is a correlation between the acid effect and the trabecular structure. The explanation is due to that all bone samples present a different microarchitecture and therefore, the decalcifying effect varied in each one. However, the morphometric quantification correlates all necessary parameters to reconstruct the acid effect in each sample as it is described in the next section.

\subsection{Quantification of the influence of TBD-1 on the bone structure}

Figure 6 shows the characterization of the trabecular bone determined through a quantitative analysis performed on the regions $\mathrm{A}$ and $\mathrm{B}$. According to the bone mineral content (BMC) percentage (Fig. 6a), samples 1, 2 and 3 present a similar microstructure since containing higher cancellous bone density than samples 4 and 5. Each microarchitecture is represented by images that were taken with a scanning electron microscope (SEM, TESCAN LYRA 3). Photographs confirm that samples 4 and 5 seem to be more porous than samples 1,2, and 3, it indicates that these are less dense. The dependency on extraction location caused a morphometric heterogeneity in the selection process of the samples. This is very common in the bone tissue since it is a graded material. For our application, there are no negative implications for the experimental tests because the calculations permitted to estimate the morphometric characteristics of each sample as well as the TBD-1 action.

Figure $6 \mathrm{~b}$ depicts the trabecular wall thickness measured on regions $\mathrm{A}$ and $\mathrm{B}$, the graph allows us to infer that trabecular walls are some homogeneous, but the connectivity and volume differ among these. This can be logically argued due to differences in bone mineral density, which means that the trabecular bone can vary the porosity levels. Table 5 lists different physical measurements before and after the decalcifying process; in which are bone mineral (BM) apparent density, removed bone fraction volume $\eta$, estimated mass submerged on TBD-1, total bone mineral content (BMC) in TBD-1, and bone mineral density variation. It is important to point out that the sample enumeration is associated with the immersion time, as described in Fig. 3. 

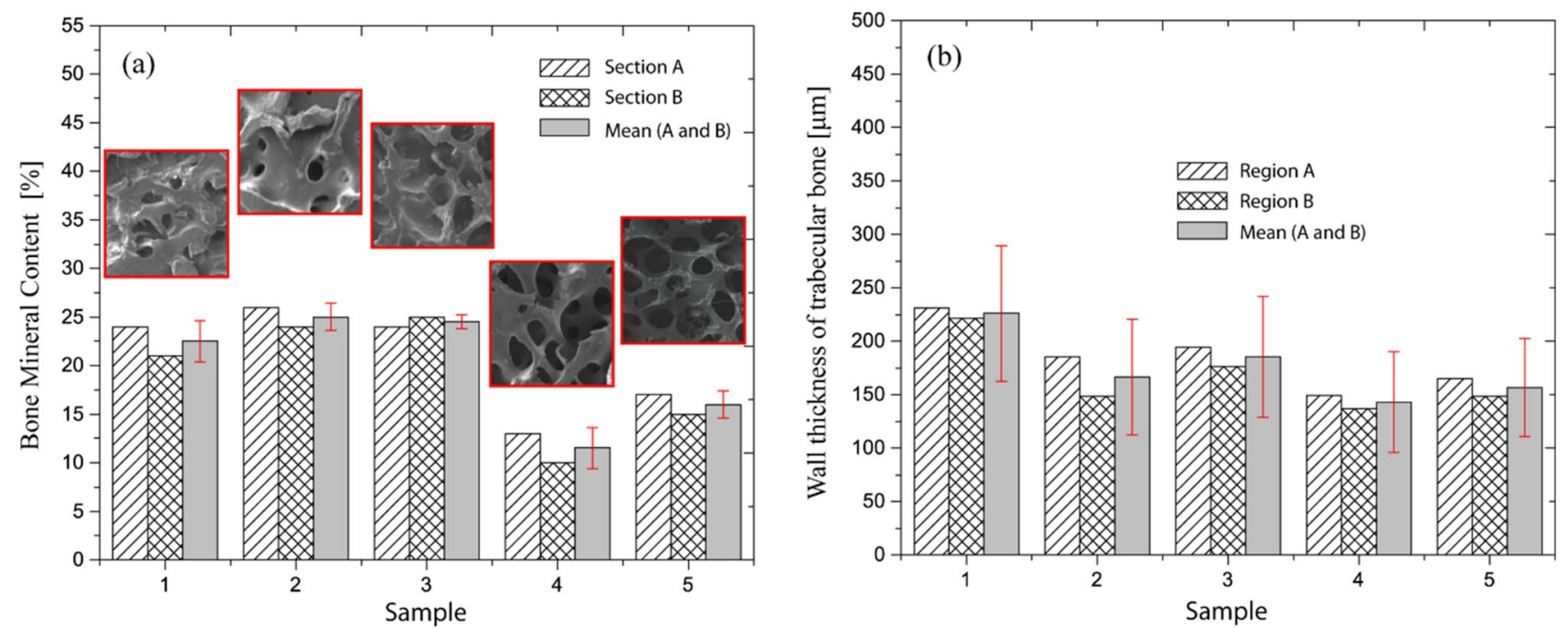

Fig. 6 Morphometry of bone samples performed the Micro-CT analysis and corresponding pristine mineral density percentage of the samples in sections $\mathrm{A}$ and $\mathrm{B}$ and its mean value

Table 5 Mass measurements before and after the decalcifying process

\begin{tabular}{|c|c|c|c|c|c|c|}
\hline Sample & $\begin{array}{l}\text { BM apparent d } \\
\left.\text { Density }\left(\mathrm{g} / \mathrm{mm}^{3}\right]\right)\end{array}$ & $\begin{array}{l}\text { Bone mass reduction } \\
(\mathrm{mg}) \text { After TBD-1 }\end{array}$ & $\begin{array}{l}\text { Removed bone volume frac- } \\
\text { tion Mean(A,B)-C (\%) } \\
\eta\end{array}$ & $\begin{array}{l}\text { Bone mass } \\
\text { immersed on } \\
\text { TBD-1 (mg) }\end{array}$ & 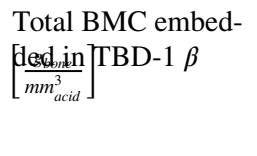 & $\begin{array}{l}\text { Bone density } \\
\text { variation by } \\
\text { TBD-1 action } \\
\eta \cdot \beta\left(\mathrm{g} / \mathrm{mm}^{3}\right)\end{array}$ \\
\hline 1 & 0.8346 & 40.3400 & 11.1111 & 363.0600 & 0.8899 & 0.0989 \\
\hline 2 & 0.4294 & 83.6800 & 12.0000 & 697.333 & 1.8513 & 0.2222 \\
\hline 3 & 0.7705 & 51.6400 & 6.1225 & 843.4531 & 2.0361 & 0.1247 \\
\hline 4 & 0.2860 & 96.1800 & 47.8261 & 201.1036 & 0.5662 & 0.2708 \\
\hline 5 & 0.3531 & 167.4200 & 37.5000 & 446.4533 & 0.7549 & 0.2831 \\
\hline
\end{tabular}

In Table 5, it is evidenced that samples 4 and 5 contain smaller values in BM apparent density, which is also an indicator that the samples are more porous. The porosity allows the acid flow easier inside the trabecular structure by capillarity. This effect produces a better effectivity of the acid since less acid volume covers a higher bone surface, as it will be discussed later. Otherwise, samples 1, 2, and 3 presented higher values of BM apparent density. The relation between bone mass reduction and removed bone fraction volume $\eta$ is described to characterize the acid effects in the samples, it defines how much bone mineral content (BMC) was eliminated during the contact time with the acid. For instance; in sample number 1; the mass reduction was $40 \mathrm{~g}$, which corresponds to $11.11 \%$ of removed BMC. In column six of Table 5; it is computed the relative density of BMC embedded in TBD-1, higher values show that acid cover less superficial area since more mass is immersed in acid. This parameter relates to the rate of bone mass reduction.

The immersed mass (IM) in TBD- 1 was estimated with the relation between $\eta$ and bone mass reduction; results indicate that the IM is most significant in sample 3, followed by samples $2,5,1$, and 4 , respectively. If these values are divided by the acid volume is determined the total BMC in TBD-1, which means that the action superficial of the acid is higher for lower values of total BMC in TBD-1. Therefore, as a consequence, it is produced a faster bone mass reduction. An explanation is given by the differences in the inner trabecular connections that allow having more or less contact with the acid substance. The results plotted in Fig. 7 synhesize the above discussion, which include an approximated model for the acid action reconstructed with the experimental data of the last column of Table 5 . The adjusted model is described in the following density equation

$\rho_{h}=B V_{\text {sub }}\left(\rho_{0}+A e^{t / t_{0}}\right)$.

where $B V_{\text {sub }}$ represent the quantity of immersed bone volume in acid. Equation (4) permits to predict the bone mass reduction under acid action TBD-1, as illustrated in Fig. 7b. In 

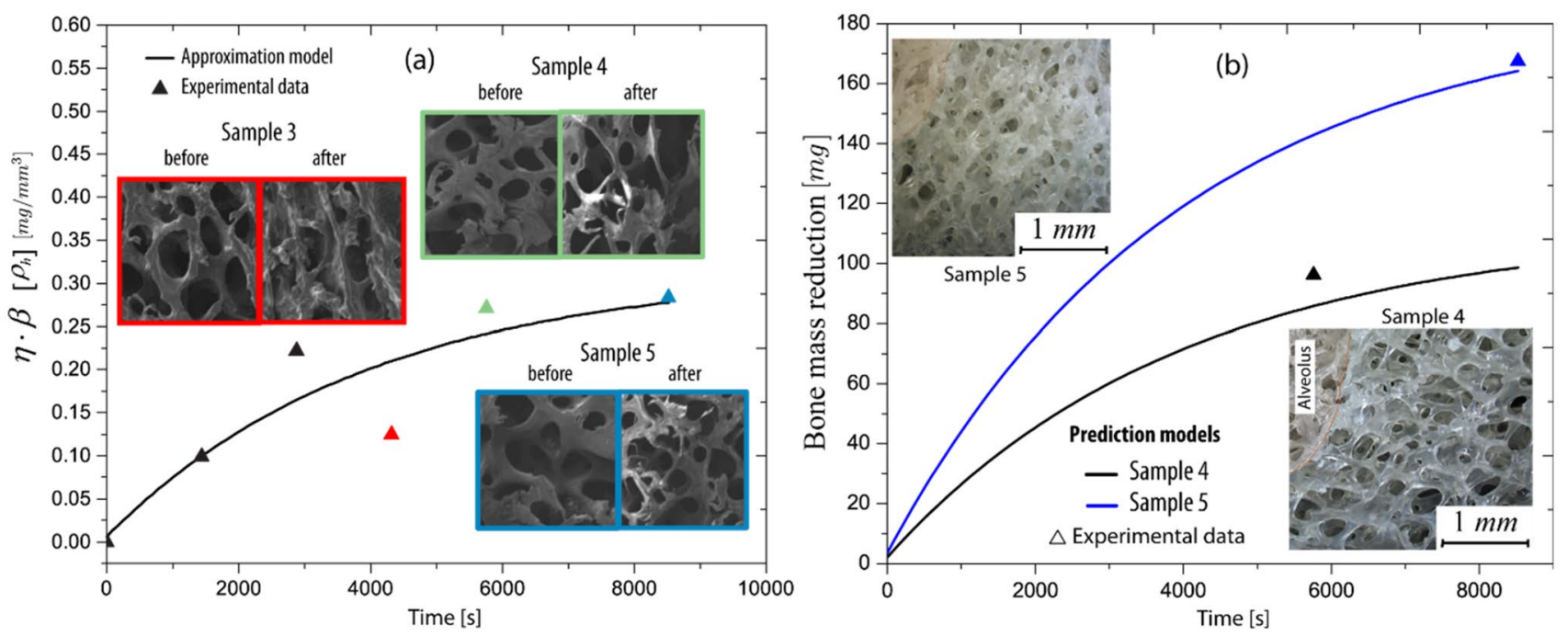

Fig. 7 a Model of the TBD-1 influence on the bone samples during the exposure time. SEM images of the samples 3, 4 and 5 before and after. b Prediction models for the bone mass reduction in samples 4 and 5

Table 6 Approximation parameters of Eq. (4)

\begin{tabular}{ll}
\hline Parameter & Values \\
\hline$\rho_{0}$ & 0.3135 \\
$A$ & -0.3075 \\
$t_{0}$ & -3965.27 \\
Chi-square & $3.84 \times 10^{-3}$ \\
Residuals $\left(\sum r^{2}\right)$ & $1.151 \times 10^{-2}$ \\
$R^{2}$ & 0.6886 \\
\hline
\end{tabular}

general terms, Fig. 7a describes the characteristic function of the reduction density $\rho_{h}$ (removed mass per cubic millimeter) caused by the immersion time in TBD-1, the parameters of Eq. (4) are listed in Table 6. It points out that the function is independent of the bone sample, but it depends on immersed bone volume. The result is important to correlate the electromechanical impedance with the bone mass reduction in a quantitative relation. SEM photographs were taken before and after the acid action to compare the changes by the bone alterations. The images were detailed for bone samples 3,4 and 5 . These illustrate the bone alterations produced by the acid since microstructures look thinner after the decalcification. Additionally, optic images were taken for samples 4 and 5 in which is detailed the bone microarchitectural after decalcification.

It is important to denote that the characterization of temporal variations in the samples is a process developed to have criteria for mass loss estimation. Then, different samples would not present any similar physical relation at the same time domain since the samples would have different density losses because of the dependency between the acid and the bone microarchitecture. This fact would not guarantee any reproducibility in the same temporal space. Therefore, we consider that the decalcifying process in five samples represented the application of the methodology (detection of mass variation), which implies that a higher number of samples would not affect the reliability of the study. For that reason, Heliscan micro-computed tomography equipment was used to avoid errors in the quantification of the mass estimation, so this method was applied in the five samples.

\subsection{Correlation of bone mass reduction through electrical resistance measurements}

Figure 8a consolidates the electrical resistance signals measured on sample 5 at different exposure times (on TBD-1) for the range $5-50 \mathrm{kHz}$. It is seen that signals presented main characteristics that were kept during the bone degradation experiment; these are associated with the trend and resonant peaks (eleven peaks marked by pink stars). According to Tinoco et al. [31] resonant peaks in the electrical resistance reflects the electrodynamics attributes of the piezo-device, which means that mechanical resonances are predominant in the electrical signal if the vibration modes deform the piezoelectric patches. In this sense, if the piezo-device is modified for any reason, the electrical impedance components will present considerable variations, as explained in Sect. 2. However, it is not the case for the carried out experimental setup since the sources of variation are due to the induced bone changes. In Fig. 8a, five observation windows were established and labeled as A, B, C, D, and E. In those frequency domains, the signals were progressively sensitive to the induced changes in the bone tissue. The intervals were selected without having a quantifiable criterion, and the selection was constrained by the constant progression of 

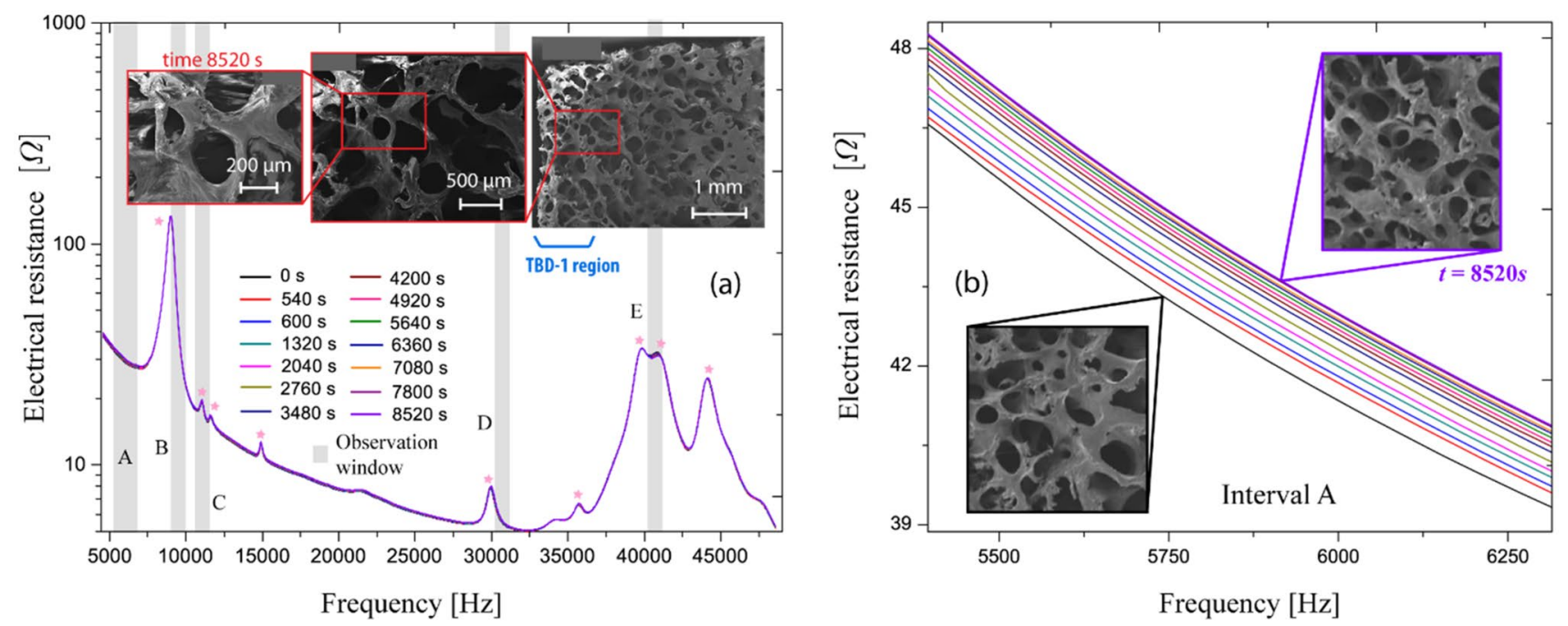

Fig. 8 a Results for electrical resistance measurements taken in the main sample (sample 5) in the frequency range 5-50 kHz from 0 to $8250 \mathrm{~s}$. SEM images at $1 \mathrm{~mm}, 500 \mu \mathrm{m}$ and $200 \mu \mathrm{m}$. b Observation window A defined in the range 5-6.3 kHz. Sample 5 SEM images are also shown

the signal, which indicates that the signal gradually shifted during the time in those regions.

On the bone sample 5, a bone image was taken with a SEM to evaluate the induced bone alterations in a qualitatively way. The image helps to compare the unaltered bone structure with the altered zone by the effect of the acid. This zone showed a considerable diminishing of the trabecular thickness; the observability of the bone microarchitecture was illustrated in two-scale stages.

Figure $8 b$ evidence that the baseline (initial state) moves upwards progressively while the acid acts on the immersed bone tissue during $8520 \mathrm{~s}$. Furthermore, the images confirm that the acid effectively affected the bone microstructure since the differences in the trabecular thickness before and after can be observed. From a diagnosis point of view, the experiment proves that induced bone mass reduction can be monitored using the teeth as probes. For the quantification of the bone mass changes during the time, RMSD index (see Eq. 3) was computed for the tracked signals in all described frequency intervals (A, B, C, D and E), as represented in Fig. 9a. As expected, RMSD index $(\varphi)$ increased with a defined trend, which exposes an exponential behavior. In the graph, it is possible to observe that the index exhibits
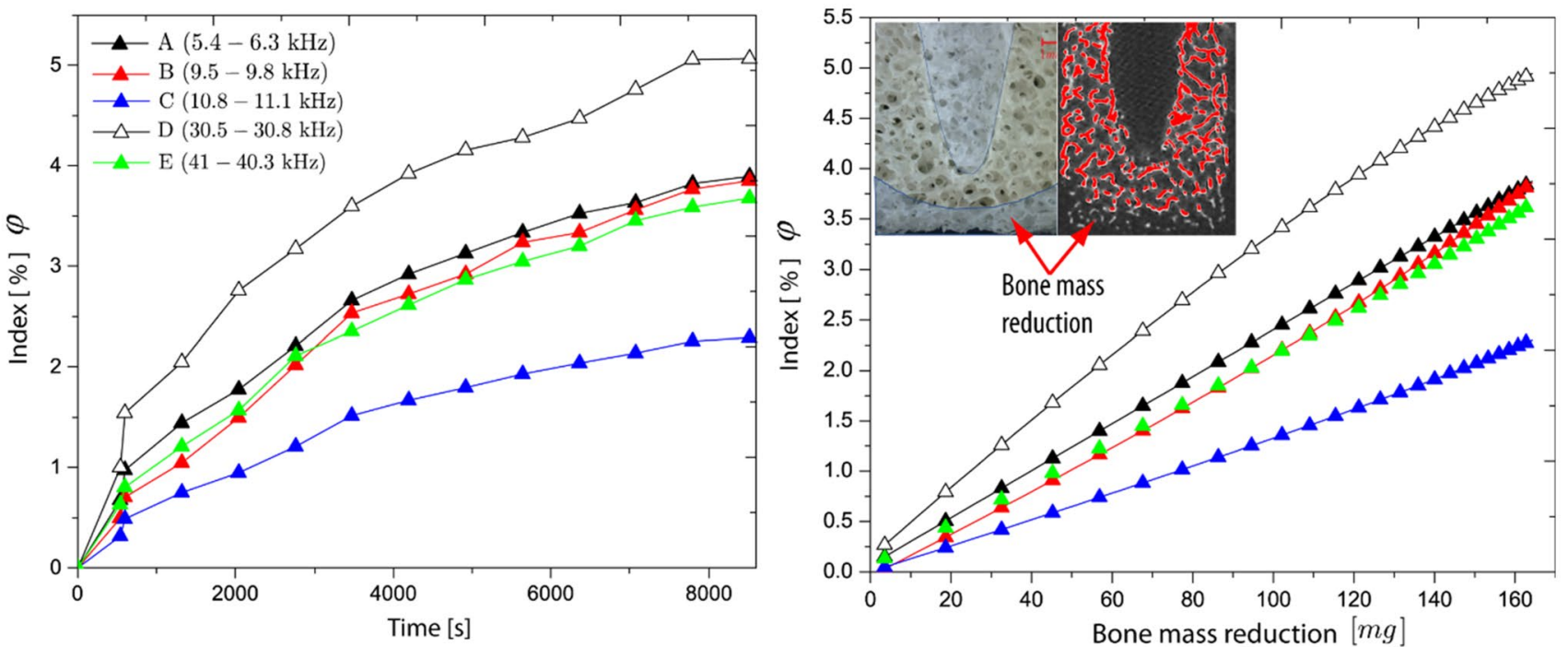

Fig. 9 a Variation of computed indexes in the time domain for the frequency intervals (A, B, C, D and E). b Correlations between electrical resistance indexes and bone mass reduction 
a similar tendency in all windows, but only three intervals showed similar values (A, B and E).

To establish a relation between $\varphi$ and the localized bone mass diminishing, both variables were plotted to correlate each estimation during the time, as illustrated in Fig. 9b. It is seen that there is an apparent linear relation between bone mass reduction and $\varphi$ obtained from electrical resistance signals. For instance, in the frequency windows A, B, and $\mathrm{E}$, there is similar behavior. This fact suggests that in these intervals, the signals present the same sensitivity to the bone alterations. In the case of the observation windows $\mathrm{C}$ and $\mathrm{D}$, these exhibit the lowest and the highest slopes, but the relation obtained for D indicates that is most sensitive for the monitoring of changes in bone density. In Fig. 9b, the image of sample 5 and the region altered by the acid TBD- 1 are compared; the reduction in its microarchitecture was modified nonhomogeneous as was explained in Sect. 3.1.

In conclusion, we can point out that the results evidence that is probable to detect alterations in the bone mass from the teeth using a vibratory device. As Fig. 9b demonstrated, bone alterations presented a quasi-linear dependence with the statistical indexes calculated from the electrical resistance. It means that from the indexes, the predictions of bone mass reduction could be estimated. As a future clinical application, the proposed methodology should be evaluated in more experimental contexts to test the viability of implementing as a diagnosis tool.

\section{Conclusions}

In the first section, a protocol of bone samples preparation and manufacturing procedures of an artificial periodontal ligament (PDL) is described to emulate a portion of a bonetooth system aiming to emulate a portion of maxillary bone. Therefore, a protocol for the manufacturing of the system bone- artificial PDL-tooth was developed, which included bone samples preparation, artificial PDL manufacturing, and assembly. In a second section, a monitoring procedure is developed utilizing a low-cost piezo-actuated device that allows observing the changes in a bone sample subjected to bone alterations induced by an acid substance.

The functionality of the piezo-actuated device was described and validated for a potential application in the biomonitoring of bone tissue changes. Localized bone alterations were detected using a human tooth as a probe in a frequency range between 5 and $50 \mathrm{kHz}$ (applying EMI technique). Results evidenced that bone density changes were read by the electrical resistance measurements; this allowed to inspect the sensitivity of the EMI technique. Besides, the experimental results showed that when the bone structure was altered gradually by the acid solution (TBD-1), the electrical resistance presented an appropriate sensitiveness since incremented its value when the sample reduced its bone mass. The quantified indexes with the electrical resistance evidenced an exponential behavior during the exposure time of the sample inside the acid. These results permitted correlating the induced bone density changes with the computed indexes. The correlation demonstrated that bone alterations present a linear dependence with the quantified statistical metric. In a real context, this will imply that after an eventual metabolic change in the bone, bone monitoring will be possible since the changes will affect the global stiffness (Young modulus or geometry changes), and therefore the device could detect it. Future works will be focused on improving the presented device to implement it in a real biomedical application since the mechanical vibrations will be a less invasive way to observe the bone alterations.

Acknowledgements This research was funded by a grant from Departamento Administrativo de Ciencia, Tecnología e Innovación (COLCIENCIAS) (Grand code 121974455599) legalized contract 739-2016 and obtained in the announcement 744, year 2016. We want to extend acknowledgements to Centro de Innovación Roldán (CIR), for their financial and technical support. Additionally, this research was carried out under the project CEITEC 2020 (LQ1601) with financial support from the Ministry of Education, Youth and Sports of the Czech Republic under the National Sustainability Programme II and Ceitec Nano+ project, CZ.02.01/0.0./.0.0./16_013/0001728 under the program OP RDE.

Financial support This study was supported by Grand Code 121974455599 from Departamento Administrativo de Ciencia, Tecnología e Innovación (COLCIENCIAS). Further, it was supported from Ministry of Education, Youth and Sports of the Czech Republic under the National Sustainability Programme II and Ceitec Nano + Poject, CZ.02.01/0.0./0.0.0./16_013/0001728.

\section{Compliance with ethical standards}

Conflicts of interest The authors declare that they do not have conflicts of interest.

Ethical approval This paper does not contain any studies with human participants or animals carried out by any of the authors.

\section{References}

1. Hernlund E, Svedbom A, Ivergård M, Compston J, Cooper C, Stenmark J, McCloskey EV, Jönsson B, Kanis JA. Osteoporosis in the European Union: medical management, epidemiology and economic burden. Arch Osteoporos. 2013;8(1-2):13.

2. Melton LJ 3rd. How many women have osteoporosis now? J Bone Miner Res. 1995;10:175-7.

3. Kanis JA, Johnell O, Oden A, Sembo I, Redlund-Johnell I, Dawson A, De Laet C, Jonsson B. Long-term risk of osteoporotic fracture in Malmö. Osteoporos Int. 2000;11(669-674):7.

4. Van Staa TP, Dennison EM, Leufkens HGM, Cooper C. Epidemiology of Fractures in England and Wales. Bone. 2001;29:517-22.

5. Kinds MB, Bartels LW, Marijnissen ACA, Vincken KL, Viergever MA, Lafeber FPJG, de Jong HWAM. Feasibility of bone density 
evaluation using plain digital radiography. Osteoarthritis Cartil. 2011;19(11):1343-8.

6. Inaba M, Ejima KI, Motoyoshi M, Arai Y, Honda K, Shimizu N. Measuring bone density at orthodontic miniscrew implantation sites using microcomputed tomography. Int J Oral Maxillofacial Implants. 2015;30(2).

7. Kanis JA, Johnell O, Gullberg BO, Allander E, Dilşen G, Gennari $\mathrm{C}$, Miravet L. Evidence for efficacy of drugs affecting bone metabolism in preventing hip fracture. BMJ. 1992;305(6862):1124-8.

8. Ward KA, Adams JE, Hangartner TN. Recommendations for thresholds for cortical bone geometry and density measurement by peripheral quantitative computed tomography. Calcif Tissue Int. 2005;77(5):275-80.

9. Chugh T, Jain AK, Jaiswal RK, Mehrotra P, Mehrotra R. Bone density and its importance in orthodontics. J Oral Biol Craniofacial Res. 2013;3(2):92-7.

10. Scheibel PC, Ramos AL, Iwaki LCV. Is there correlation between alveolar and systemic bone density? Dental Press J Orthodontics. 2013;18(5):78-83.

11. López López J, Estrugo-Devesa A, Jané Salas E, Ayuso Montero R, Gómez Vaquero C. Early diagnosis of osteoporosis by means of orthopantomograms and oral x-rays: a systematic review. Medicina Oral, Patología Oral y Cirugia Bucal. 2011;16(7):905-13.

12. Li HN, Ren L, Jia ZG, Yi TH, Li DS. State-of-the-art in structural health monitoring of large and complex civil infrastructures. J Civ Struct Health Monit. 2016;6:3-16.

13. Huo L, Wang B, Chen D, Song G. Monitoring of pre-load on rock bolt using piezoceramic-transducer enabled time reversal method. Sensors. 2017;17:2467.

14. Wang B, Huo L, Chen D, Li W, Song G. Impedance-based prestress monitoring of rock bolts using a piezoceramic-based smart washer-a feasibility study. Sensors. 2017;17:250.

15. Tinoco HA, Gomez JP, Torres J, Velasco MA. Structural differentiation of tooth supporting substances with the electromechanical impedance technique. Int J Mech Mechatron Eng. 2016;16:8-15.

16. Na W, Baek J. A review of the piezoelectric electromechanical impedance based structural health monitoring technique for engineering structures. Sensors. 2018;18:1307.

17. Martinez-Luengo M, Kolios A, Wang L. Structural health monitoring of offshore wind turbines: A review through the Statistical Pattern Recognition Paradigm. Renew Sustain Energy Rev. 2016;64:91-105.

18. Liang C, Sun FP, Rogers CA. An impedance method for dynamic analysis of active material systems. J Vib Acoust. 1994;116(1):120-8.

19. Park G, Inman DJ. Structural health monitoring using piezoelectric impedance measurements. Philos Trans Roy Soc A Math Phys Eng Sci. 2006;365(1851):373-92.

20. Yang Y, Lim YY, Soh CK. Practical issues related to the application of the electromechanical impedance technique in the structural health monitoring of civil structures: I. Exp Smart Mater Struct. 2008;17(3):035008.

21. Annamdas VGM, Soh CK. Application of electromechanical impedance technique for engineering structures: review and future issues. J Intell Mater Syst Struct. 2010;21(1):41-59.

22. Bhalla S, Suresh R. Condition monitoring of bones using piezotransducers. Meccanica. 2013;48(9):2233-44.

23. Khan M, Sirdeshmukh SPSMA, Javed K. Evaluation of bone fracture in animal model using bio-electrical impedance analysis. Perspect Sci. 2016;8:567-9.

24. Srivastava S, Bhalla S, Madan A. Shape memory alloy actuation of non-bonded piezo sensor configuration for bone diagnosis and impedance based analysis. Biomed Eng Lett. 2019;1-13.

25. Srivastava S, Bhalla S, Madan A. Assessment of human bones encompassing physiological decay and damage using piezo sensors in non-bonded configuration. J Intell Mater Syst Struct. 2017;28(14):1977-92.

26. Srivastava S, Bhalla S. Numerical evaluation of nonbonded piezo sensor for biomedical diagnostics using electromechanical impedance technique. Int J Numer Methods Biomed Eng. 2019;35(2):e3160.

27. Tabrizi A, Rizzo P, Ochs MW. Electromechanical impedance method to assess dental implant stability. Smart Mater Struct. 2012;21(11):115022.

28. Ribolla ELM, Rizzo P, Gulizzi V. On the use of the electromechanical impedance technique for the assessment of dental implant stability: modeling and experimentation. J Intell Mater Syst Struct. 2015;26(16):2266-80.

29. Ponder RI, Safaei M, Anton SR. Validation of impedance-based structural health monitoring in a simulated biomedical implant system. In: ASME 2018 Conference on Smart Materials, Adaptive Structures and Intelligent Systems, 2018 (pp. V002T05A008V002T05A008). American Society of Mechanical Engineers.

30. Tinoco HA, Robledo-Callejas L, Marulanda DJ, Serpa AL. Damage detection in plates using the electromechanical impedance technique based on decoupled measurements of piezoelectric transducers. J Sound Vib. 2016;384:146-62.

31. Tinoco H, Cardona C, Peña F, Gomez J, Roldan-Restrepo S, Velasco-Mejia M, Barco D. Evaluation of a piezo-actuated sensor for monitoring elastic variations of its support with impedancebased measurements. Sensors. 2019;19(1):184.

32. Xie X, Kolthoff N, Bärenholt O, Nielsen SP. Validation of a legto-leg bioimpedance analysis system in assessing body composition in postmenopausal women. Int J Obesity. 1999;23(10):1079.

33. Pietrobelli A, Rubiano F, St-Onge MP, Heymsfield SB. New bioimpedance analysis system: improved phenotyping with wholebody analysis. Eur J Clin Nutr. 2004;58(11):1479.

34. Balmer TW, Ansó J, Muntane E, Gavaghan K, Weber S, Stahel A, Büchler P. In-Vivo electrical impedance measurement in mastoid bone. Ann Biomed Eng. 2017;45(4):1122-32.

35. Kimel-Naor S, Abboud S, Arad M. Parametric electrical impedance tomography for measuring bone mineral density in the pelvis using a computational model. Med Eng Phys. 2016;38(8):701-7.

36. Safaei M, Nolan EC, Anton SR. Finite element evaluation of EMI-based structural health monitoring in high frequencies. In: Health Monitoring of Structural and Biological Systems XIII, 2019; (Vol. 10972, p. 109720C). International Society for Optics and Photonics.

37. Rodríguez-Palomo D, Ramírez-Zamora J. Técnica de conservación de huesos en peróxido de hidrógeno. Medicina Legal de Costa Rica. 2009;26(2):117-23.

38. Mairs S, Swift B, Rutty GN. Detergent: an alternative approach to traditional bone cleaning methods for forensic practice. Am J Forensic Med Pathol. 2004;25(4):276-84.

39. Gomez JP, Peña FM, Martínez V, Giraldo DC, Cardona CI. Initial force systems during bodily tooth movement with plastic aligners and composite attachments: a three-dimensional finite element analysis. Angle Orthodontist. 2014;85(3):454-60.

40. Rees JS. An investigation into the importance of the periodontal ligament and alveolar bone as supporting structures in finite element studies. J Oral Rehabil. 2001;28(5):425-32.

41. Jeon PD, Turley PK, Ting K. Three-dimensional finite element analysis of stress in the periodontal ligament of the maxillary first molar with simulated bone loss. Am J Orthod Dentofac Orthop. 2001;119(5):498-504.

42. Yan W, Chen WQ. Structural health monitoring using high-frequency electromechanical impedance signatures. Adv Civil Eng. 2010; $1-11$

43. Sirohi J, Chopra I. Fundamental understanding of piezoelectric strain sensors. J Intell Mater Syst Struct. 2000;11(4):246-57. 
44. Barco DR, Tinoco HA, Cardona CI, Peña FM. Piezo-actuated device for a bio-structural monitoring application through vibration-based condition and electromechanical impedance measurement. Aug/2019. In: Proceedings of 2nd International Conference on Numerical Modelling in Engineering, Beijing, China, 2019; pp. $1-7$.

45. Ribolla EL, Rizzo P. Modeling the electromechanical impedance technique for the assessment of dental implant stability. J Biomech. 2015;48(10):1713-20.

46. Farrar CR, Doebling SW, Nix DA. Vibration-based structural damage identification. Philos Trans Roy Soc Lond A Math Phys Eng Sci. 2001;359(1778):131-49.

47. Tinoco HA, Marulanda DJ. Damage identification in active plates with indices based on Gaussian confidence ellipses obtained of the electromechanical admittance. J Nondestr Eval. 2015;34(3):1-16.

48. Tawie R, Lee HK. Monitoring the strength development in concrete by EMI sensing technique. Constr Build Mater. 2010;24(9):1746-53.
49. Bouxsein ML, Boyd SK, Christiansen BA, Guldberg RE, Jepsen KJ, Müller R. Guidelines for assessment of bone microstructure in rodents using micro-computed tomography. J Bone Miner Res. 2010;25(7):1468-86.

50. Calvo-Guirado JL, Ramírez-Fernández MP, Gómez-Moreno G, Maté-Sánchez JE, Delgado-Ruiz R, Guardia J, Bravo LA. Melatonin stimulates the growth of new bone around implants in the tibia of rabbits. J Pineal Res. 2010;49(4):356-63.

Publisher's Note Springer Nature remains neutral with regard to jurisdictional claims in published maps and institutional affiliations. 